\title{
Burden of Cardiovascular Disease in Adult Patients with Type 1 Diabetes in the US
}

\author{
Steve Edelman ${ }^{1} \cdot$ Fang Liz Zhou $^{2} \cdot$ Ronald Preblick $^{2} \cdot$ Sumit Verma ${ }^{3} \cdot$ Sachin Paranjape ${ }^{2} \cdot$ Michael J. Davies $^{4}$. \\ Vijay N. Joish ${ }^{4}$
}

Published online: 29 January 2020

(c) The Author(s) 2020

\begin{abstract}
Background and objectives The burden imposed by cardiovascular disease (CVD) on patients with type 1 diabetes (T1D) in the US has not been thoroughly addressed. In a retrospective observational analysis of the Optum ${ }^{\circledR} \mathrm{Clinformatics}^{\mathrm{TM}}$ Data Mart database, the prevalence of CVD and cardiovascular risk factors (CVRF) as well as health economic outcomes were evaluated in adults with T1D.

Methods Patients with at least one T1D medical claim between January 1, 2016, and December 31, 2016, were divided into cohorts based on the presence of CVD and/or CVRF. Descriptive and multivariate analyses enabled comparisons of healthcare resource utilization and costs between the cohorts.

Results The analysis included 12,687 patients: CVD, 2871; CVRF, 5371; and no CVD/CVRF, 4445. The period prevalence of CVD and CVRF in the combined baseline and follow-up periods was $27 \%$ and $44 \%$, respectively. Fewer patients in the no-CVD/CVRF cohort had a claim of a diabetes-related inpatient admission compared with the CVD cohort (8\% vs. 26\%, respectively; $P<0.001$, standardized mean difference [SMD] $>0.1$ ). Likewise, fewer patients with no CVD/CVRF visited the emergency department vs. those with CVRF or CVD (diabetes-related: $4 \%$ vs. $7 \%$ and $18 \%$, respectively; $P<0.001$, SMD $>0.1)$. Higher overall costs were observed for the CVD and CVRF vs. the no-CVD/CVRF cohort $(\$ 30,241$ and $\$ 16,220$, respectively, vs. $\$ 11,761 ; P<0.05$ and $\mathrm{SMD} \geq 0.1$ for both).

Conclusions Cardiovascular comorbidities are common among US adults with T1D. Considering their significant economic burden, optimal management is of the utmost importance to improve patient outcomes and reduce healthcare costs.
\end{abstract}

\section{Introduction}

Very poor glycemic control has been associated with a tenfold-increased risk of death from cardiovascular disease (CVD) in type 1 diabetes (T1D) compared with the general population [1]. Patients with T1D also show an

Electronic supplementary material The online version of this article (https://doi.org/10.1007/s41669-019-00192-9) contains supplementary material, which is available to authorized users.

Steve Edelman

svedelman@vapop.ucsd.edu

1 Veterans Affairs Medical Center, University of California San Diego, 990 Highland Drive, Suite 312, Solana Beach, CA 92075, USA

2 Sanofi US, Inc., Bridgewater, NJ, USA

3 Evidera, Bethesda, MD, USA

4 Lexicon Pharmaceuticals, Inc., Basking Ridge, NJ, USA approximately twofold greater incidence of hospitalization due to heart failure than people without diabetes; advanced age and diabetes duration, presence of obesity and renel impairment, as well as history of smoking and prior ischemic heart disease and stroke are good predictors of increased risk for this heart condition [2].

Various risk factors with a significant negative impact on the prevalence of CVD among patients with T1D have been identified throughout the years, namely hypertension, proteinuria, obesity, dysglycemia, dyslipidemia, tobacco use, advanced age, and longer duration [3]. Recent medical advances resulting in decreased mortality from renal impairment and acute metabolic complications have also contributed to an increase in the number of patients with T1D who are exposed to age-related cardiovascular risk factors (CVRF) and CVD [4].

The epidemiological and disease burden estimates of CVRF and CVD in T1D have commonly been extrapolated from studies conducted in type 2 diabetes (T2D) or 


\section{Key Points for Decision Makers}

Considering the worldwide increase in the prevalence of conditions such as obesity and hypertension, patients with type 1 diabetes (T1D) often present with concomitant cardiovascular disease (CVD) and cardiovascular risk factors (CVRF), which can significantly increase the economic burden of disease.

This analysis of a large health insurance claims database in the US revealed increased healthcare resource utilization and costs among adult patients with T1D and CVD, and/or CVRF compared with those with neither.

Management of cardiovascular comorbidities constitutes an important component of care for adults with T1D; optimal management is critical to improve patient outcomes and reduce healthcare costs.

general diabetes populations [3], but there are differences in the age of onset, duration, and natural history of CVD between patients with T1D and T2D, as well as variations in the pathogenesis of cardiovascular conditions such as atherosclerosis $[4,5]$.

The incidence of coronary heart disease for men and women with T1D aged 20-40 years was determined to be approximately 5 and 11 times, respectively, the rates for individuals without diabetes [6]. The excess risk of mortality and CVD increases with decreasing age of onset of T1D, and is higher for women [5]. In fact, the estimated life expectancy at 20 years of age is reduced by 11 years for men and almost 13 years for women with T1D compared with the general population, and approximately one-third of the excess risk of death is due to CVD [7]. However, most of these large recent studies of CVD in patients with T1D have used data collected from European registries [1, 5-9]. In the US, the Pittsburgh Epidemiology of Diabetes Complications study revealed that CVD was responsible for $40 \%$ of the deaths among patients with T1D for $>20$ years [10], but there is a paucity of data on the impact of the cardiovascular burden on diabetes-related and all-cause health economic outcomes in the US.

It is particularly important for population health managers to have comprehensive and up-to-date data that elucidate the cardiovascular burden among patients with T1D. To meet this current gap in knowledge, the present analysis evaluated health insurance claims from a large database-the Optum ${ }^{\circledR}$ Clinformatics ${ }^{\mathrm{TM}}$ Data Mart database, which comprises approximately 17 million annual covered lives-to evaluate the real-world prevalence of and disease burden (as measured by health economic outcomes) associated with CVRF and CVD in T1D in the US.

\section{Methods}

\subsection{Design and Eligibility Criteria}

In a retrospective analysis of healthcare insurance claims, data from the Optum ${ }^{\circledR}$ Clinformatics ${ }^{\mathrm{TM}}$ Data Mart database were collected from January 1, 2015, to December 31, 2017, and analyzed. Optum ${ }^{\circledR}$ Clinformatics ${ }^{\mathrm{TM}}$ Data Mart is a database of administrative health claims for members of a large managed care company affiliated with OptumInsight [11]. It includes approximately 17 million annual covered lives, based on enrollment in a UnitedHealth insurance plan as of June 2018, for a total of approximately 60 million covered lives over an 11 -year period. It comprises commercial health plan data, but also includes historic claims for managed Medicaid and Medicare Plus Choice members; the population covered is geographically diverse, spanning all 50 states in the US. In addition to medical claims, pharmacy claims, and laboratory results, the database includes information on inpatient stays, standard pricing, and member eligibility data. All data are de-identified prior to acquisition [11].

This analysis included patients with at least one T1D medical claim during the identification period from January 1, 2016, to December 31, 2016; the date of first medical claim with a diagnosis code for T1D in the identification period was defined as the index date. The diagnosis of diabetes was classified as T1D, according to a validated algorithm developed by Klompas et al. [12] using the combination of diabetes diagnosis codes and antihyperglycemic treatment pattern in T1D. The positive predictive value for the Klompas algorithm was $88 \%$ in the original publication [12] and $94.5 \%$ in an external validation study [13].

Patients had to be $\geq 18$ years at the index date and have continuous eligibility (without gaps) for 12 months prior to the index date and until the earliest occurrence of death (based on data from the Social Security Administration) or the end of the 12-month period after the index date or December 31, 2017, while maintaining continuous enrollment. Patients were excluded if they met any of the following criteria: (1) patients with follow-up $<30$ days post-index; (2) women who were pregnant at any time during the 12-month baseline period; and (3) no prescription claim for rapid-acting or short-acting insulin within 6 months prior to the index date.

Patients were divided into three cohorts based on baseline presence of CVD and/or CVRF [details on International Classification of Diseases (ICD) [14] codes are provided in the Electronic supplementary material, ESM]. The CVD cohort included patients with CVD, defined as $\geq 1$ medical claim for myocardial infarction, stroke 
(ischemic or hemorrhagic), coronary heart disease, heart failure, cardiac arrhythmia (including atrial fibrillation or flutter), pulmonary embolism, or peripheral vascular disease; patients in this cohort could also have CVRF in the presence of CVD. The CVRF cohort included patients with CVRF, defined as $\geq 1$ medical claim of hypertension and $\geq 1$ prescription for an antihypertensive agent, or $\geq 1$ medical claim for hypercholesterolemia or hyperlipidemia and $\geq 1$ prescription for a lipid-modifying medication, or $\geq 1$ medical claim for chronic kidney disease (CKD), or $\geq 1$ medical claim for obesity, but without CVD (details on diagnosis codes and National Drug Codes are provided in the ESM). Finally, the no-CVD/CVRF cohort included patients with no medical claims for CVD or CVRF, as defined above. This classification was strictly based on information captured during the baseline period.

Patients were followed for a maximum of 12 months after the index date. The analyses were carried out using the Python and R programming languages on Apache Spark and in accordance with a prespecified protocol, following the principles, regulations, and guidelines governing medical practice and ethics in the US for noninterventional studies and/or observational studies. No Institutional Review Board approval was required.

\subsection{Outcome Variables}

Patient baseline demographic data, including age, gender, health plan type, and geographic region, were collected at the index date. The period for assessing the prevalence of CVRF and CVD was from 12 months prior to the index date until the end of the follow-up period.

All-cause and diabetes-related healthcare resource utilization data were collected during the follow-up period. Healthcare resource utilization data included prescription medications, emergency department (ED) visits, inpatient admissions, inpatient length of stay (days), and outpatient physician office visits. Because not all patients were expected to have 12 months of follow-up, annualized all-cause and diabetes-related healthcare costs per patient were reported for inpatient admissions, ED visits, outpatient physician office visits, outpatient services, and prescription medications. Diabetes-related healthcare utilization and costs were defined as resource use and cost on medical claims with a primary or secondary diagnosis of diabetes and antihyperglycemic medications. All costs were inflation adjusted to 2018 US dollars using the annual medical care component of the Consumer Price Index [15] to account for inflation from the time the cost was incurred to the end date of the analysis, which allowed for the direct comparison of costs during the analysis period.

\subsection{Statistical Analyses}

Descriptive statistical and univariate analyses were performed for prevalence and healthcare resource utilization and cost data. Differences between cohorts were assessed using chi-square tests for categorical variables and $t$ tests for continuous variables; for the age variable, an overall $F$ test was used to detect the presence of any differences among groups. Fisher's exact tests and/or nonparametric tests (e.g., the Wilcoxon rank-sum test for skewed data such as resource utilization/costs) were used as appropriate. Significant differences were determined by calculating $P$ values and standardized mean differences (SMDs), defined as the absolute difference in sample means divided by an estimate of the pooled standard deviation of the variable. Significant differences between variables had to have $P<0.05$ and SMD $>0.1$.

The differences between groups with no CVD/CVRF and with CVRF or CVD were analyzed with a generalized linear model (GLM) that allowed for variation in both the link and the family of distribution and was based on the response variable for resource utilization-a GLM with a Poisson distribution and log link for count outcomes, a twopart model using logistic regression for the binomial component, and a standard overall least squares (OLS) model for cost outcomes. Multivariate analyses were adjusted for confounding variables that were found to be significantly different between patients among all cohorts in the univariate analysis, i.e., age, ethnicity, gender, health insurance plan type (commercial vs. Medicare), geographical region, modified Deyo-Charlson Comorbidity Index (DCI), and comorbidities (i.e., urinary tract infection, hypothyroidism, and depressive disorder). Poisson, quasi-Poisson, and negative binomial models were compared according to the Akaike information criterion and likelihood ratio test in order to select the most appropriate regression model [16]. All statistical tests conducted were two-sided with a significance level of $\alpha=0.05$. Patients with T1D who met the study eligibility criteria were included in the final analysis.

\section{Results}

\subsection{Baseline Demographic and Clinical Characteristics}

A total of 12,687 patients with T1D were included in the analysis (see Fig. 1): 2871 (23\%) in the CVD cohort; $5371(42 \%)$ in the CVRF cohort; and $4445(35 \%)$ in the no-CVD/CVRF cohort. A total of 94 patients died during the 12-month follow-up period; all the other patients had a follow-up of 365 days. 


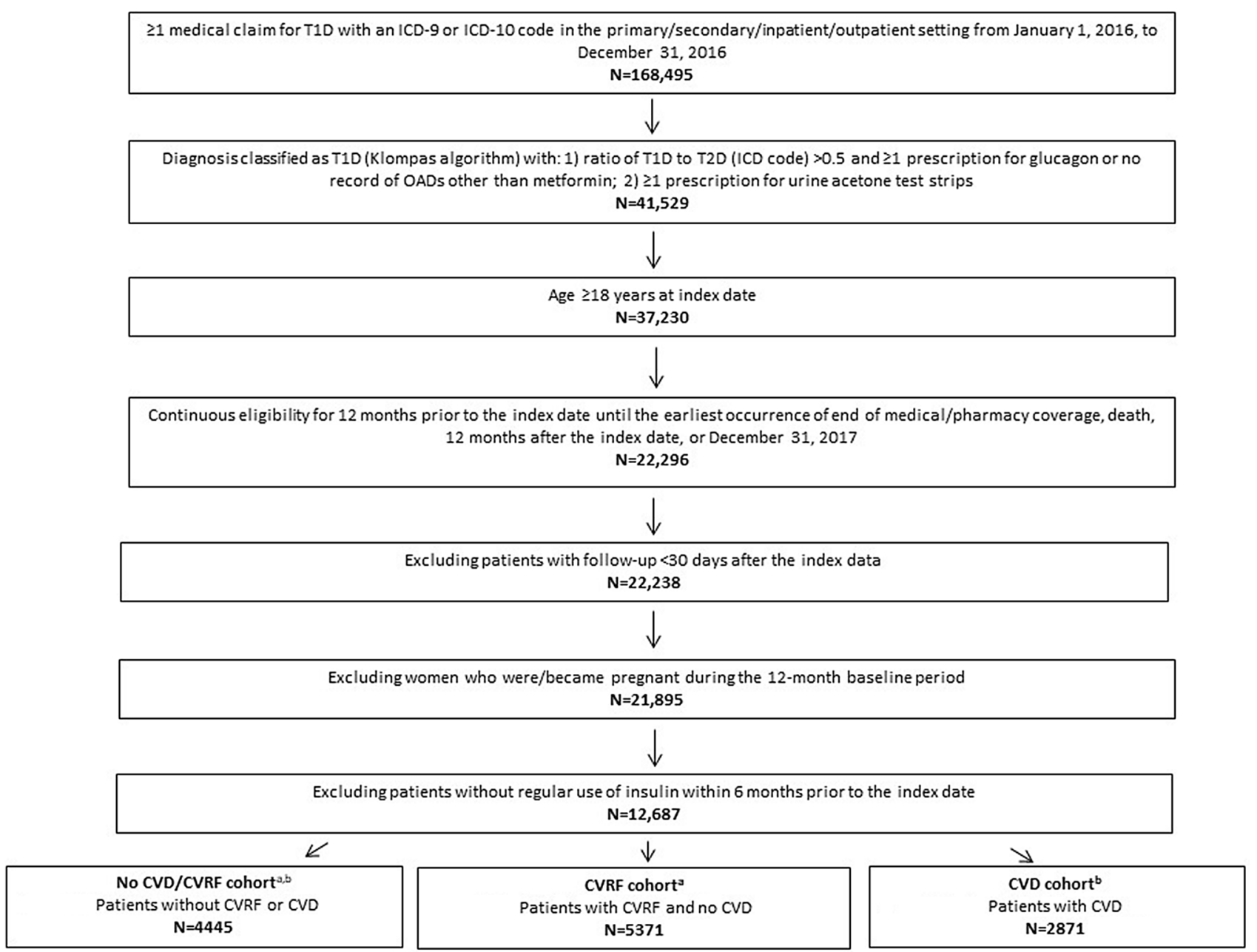

Fig. 1 Patient attrition. $C V D$ cardiovascular disease, $C V R F$ cardiovascular risk factors, $I C D$ International Classification of Diseases, $O A D$, oral antidiabetes drug, $T 1 D$, type 1 diabetes, $T 2 D$, type 2 diabetes. ${ }^{a} \mathrm{CVRF}$, including hypertension, hypercholesterolemia, hyperlipi-

The baseline demographic and clinical characteristics are summarized in Table 1. Patients in the no-CVD/ CVRF cohort were significantly younger than those in the CVRF and CVD cohorts (mean ages of 35.8 vs. 53.0 and 62.1 years, respectively; $P<0.001, \mathrm{SMD}>0.1)$. The majority of the patients in all three cohorts were white $(\geq 72 \%)$. Approximately one-third were located in the Midwest, and more than one-third were in the South.

Fewer patients in the no-CVD/CVRF vs. the CVRF and the CVD cohorts were covered by Medicare plan (5\% vs. $23 \%$ and $50 \%$, respectively; $P<0.001$ and SMD $>0.1$ for the CVRF and CVD cohorts vs. the no-CVD/CVRF cohort). The most frequent comorbidity in all three cohorts was hypothyroidism ( $21 \%$ vs. $32 \%$ and $39 \%$, respectively, for the no-CVD/CVRF, CVRF, and CVD cohorts; $P<0.001$ and SMD $>0.1$ for both CVRF and CVD vs. no CVD/CVRF), followed by urinary tract infection (4\% vs. $6 \%$ and $12 \%$, demia, chronic kidney disease, and obesity. ${ }^{\mathrm{b}} \mathrm{CVD}$, including myocardial infarction, stroke (ischemic or hemorrhagic), heart failure, cardiac arrhythmia, atrial fibrillation or flutter, coronary heart disease, pulmonary embolism, and peripheral vascular disease

respectively, for the no-CVD/CVRF, CVRF, and CVD cohorts; $P<0.001$ and SMD $>0.1$ for CVRF vs. no CVD/ CVRF) (see Table 1).

\subsection{Prevalence of CVD and CVRF}

The period prevalence of CVD (combined baseline and follow-up periods) was $27 \%(3475 / 12,687)$, which included patients who developed the condition(s) during the followup period. The proportions of different types of CVD for patients in this cohort were: cerebrovascular disease, $13 \%$; heart failure, $27 \%$; peripheral vascular disease, $43 \%$; prior myocardial infarction, coronary artery disease (CAD), and angina, $46 \%$; and cardiac arrhythmia, $50 \%$. The period prevalence of CVRF was $44 \%(5603 / 12,687)$, and the CVRF reported were CKD (13\%), obesity (22\%), hypertension and $\geq 1$ prescription for an antihypertensive agent (67\%), and 
Table 1 Demographic and clinical characteristics by diagnosis category

\begin{tabular}{|c|c|c|c|c|c|}
\hline & $\begin{array}{l}\text { No-CVD/CVRF } \\
\text { cohort }(n=4445)\end{array}$ & $\begin{array}{l}\text { CVRF } \\
\text { cohort } \\
(n=5371)\end{array}$ & $\begin{array}{l}P \text { value/SMD (CVRF } \\
\text { vs. no-CVD/CVRF } \\
\text { cohort) }\end{array}$ & CVD cohort $(n=2871)$ & $\begin{array}{l}P \text { value/SMD (CVD vs. } \\
\text { no-CVD/CVRF cohort) }\end{array}$ \\
\hline Age in years, mean (SD) & $35.8(14.2)$ & $53.0(14.4)$ & $<0.001 / 1.197 *$ & $62.1(13.9)$ & $<0.001 / 1.871^{*}$ \\
\hline Women, $n(\%)$ & $2178(49)$ & $2611(49)$ & $0.617 / 0.021$ & $1369(48)$ & $0.282 / 0.026$ \\
\hline Ethnicity, $n(\%)$ & & & $0.002 / 0.084$ & & $<0.001 / 0.131^{*}$ \\
\hline African American & $236(5)$ & $333(6)$ & & $236(8)$ & \\
\hline Asian & $81(2)$ & $87(2)$ & & $37(1)$ & \\
\hline Hispanic & $246(6)$ & $234(4)$ & & $132(5)$ & \\
\hline White & $3195(72)$ & $3977(74)$ & & $2059(72)$ & \\
\hline Other/unknown & $687(16)$ & $740(14)$ & & $407(14)$ & \\
\hline Geographic region, $n(\%)$ & & & $0.003 / 0.081$ & & $<0.001 / 0.118^{*}$ \\
\hline Northeast & $434(10)$ & $505(9)$ & & $328(11)$ & \\
\hline Midwest & $1354(31)$ & $1622(30)$ & & $771(27)$ & \\
\hline South & $1614(36)$ & $2131(40)$ & & $1162(41)$ & \\
\hline West & $1035(23)$ & $1105(21)$ & & $605(21)$ & \\
\hline Unknown & $8(0.2)$ & $8(0.1)$ & & $5(0.2)$ & \\
\hline Health insurance plan, $n(\%)$ & & & $<0.001 / 0.531^{*}$ & & $<0.001 / 1.154^{*}$ \\
\hline Commercial & $4217(95)$ & $4138(77)$ & & $1444(50)$ & \\
\hline Medicare & $228(5)$ & $1233(23)$ & & $1427(50)$ & \\
\hline Modified $\mathrm{DCI}^{\mathrm{a}}$, mean $(\mathrm{SD})$ & $0.01(0.2)$ & $0.03(0.3)$ & $<0.001 / 0.102 *$ & $0.32(1)$ & $<0.001 / 0.410^{*}$ \\
\hline \multicolumn{6}{|l|}{ Top 5 comorbidities $^{\mathrm{b}}, n(\%)$} \\
\hline Urinary tract infection & $178(4)$ & $338(6)$ & $<0.001 / 0.104^{*}$ & $350(12)$ & $<0.001 / 0.303^{*}$ \\
\hline Depressive disorder & $172(4)$ & $321(6)$ & $<0.001 / 0.097$ & $349(12)$ & $<0.001 / 0.309 *$ \\
\hline $\begin{array}{l}\text { Acute upper respiratory infec- } \\
\text { tion }\end{array}$ & $684(15)$ & $692(13)$ & $<0.001 / 0.072$ & $414(14)$ & $0.258 / 0.027$ \\
\hline Hypothyroidism & $928(21)$ & $1710(32)$ & $<0.001 / 0.251^{*}$ & 1129 (39) & $<0.001 / 0.410^{*}$ \\
\hline Acute sinusitis & $225(5)$ & $311(6)$ & $0.114 / 0.032$ & $173(6)$ & $0.076 / 0.042$ \\
\hline
\end{tabular}

$C V D$ cardiovascular disease, $C V R F$ cardiovascular risk factors, DCI Deyo-Charlson Comorbidity Index, ICD, International Classification of Diseases, $S D$ standard deviation, $S M D$ standardized mean difference

* Significant difference $P \leq 0.001$ and $\mathrm{SMD} \geq 0.1$

${ }^{a}$ The DCI was modified to exclude CVRF or CVD or diabetes-related conditions and calculated for each patient included in the study

${ }^{b}$ Based on frequency of ICD medical claims (inpatient or outpatient) identified in the no-CVD/CVRF cohort

hypercholesterolemia or hyperlipidemia and $\geq 1$ prescription for a lipid-modifying drug (74\%).

\subsection{Healthcare Resource Utilization}

The presence of CVD/CVRF and CVD significantly increased all-cause and diabetes-related resource utilization per patient per year (see Table 2). The proportion of patients with all-cause and diabetes-related inpatient admission was significantly lower in the no-CVD/CVRF vs. the CVD cohort (all-cause: $8 \%$ vs. $27 \% ; P<0.001$ and SMD $>0.1$ ), and the length of hospitalization was also shorter for the noCVD/CVRF vs. the CVD cohort (all-cause: 0.5 vs. 4.9 days; $P<0.001$ and SMD $>0.1$ ). Fewer patients in the no-CVD/ CVRF cohort had all-cause and diabetes-related ED visits vs. the CVRF and CVD cohorts (all-cause: $10 \%$ vs. $14 \%$ and $26 \%$, respectively; $P<0.001$ and SMD $>0.1$ ). Likewise, the mean number of outpatient physician visits was lower for patients in the no-CVD/CVRF vs. the CVRF and CVD cohorts. Patients with CVD or with CVRF had significantly more all-cause prescription claims compared with patients with no CVD/CVRF (64.7, 42.3 vs. 25.9, respectively; all $P<0.001$ and SMD $>0.1$ ) (see Table 2).

Healthcare costs per patient per year for the different cohorts are shown in Fig. 2. The all-cause and diabetesrelated costs associated with inpatient admission were lower for patients with no CVD/CVRF vs. CVRF and CVD $(P<0.001$ and SMD $>0.1$ for both cohorts vs. the no-CVD/ CVRF cohort). All-cause pharmacy costs were significantly higher for patients with CVRF and CVD compared with those with no CVD/CVRF, but diabetes-related pharmacy costs were only significantly higher for the CVRF cohort vs. the no-CVD/CVRF cohort. The same trend was observed for total costs, which included medical (outpatient, inpatient, and 


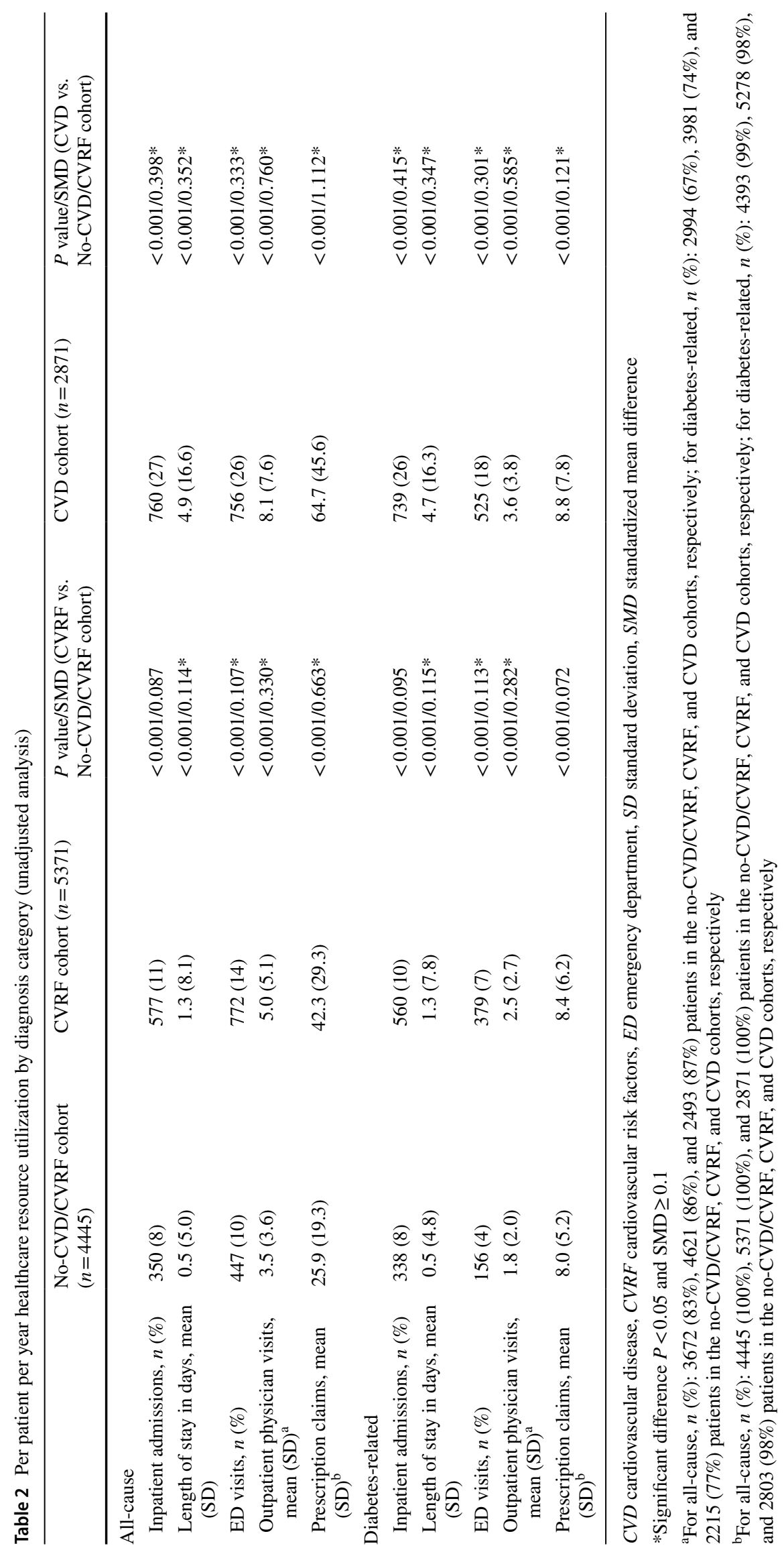




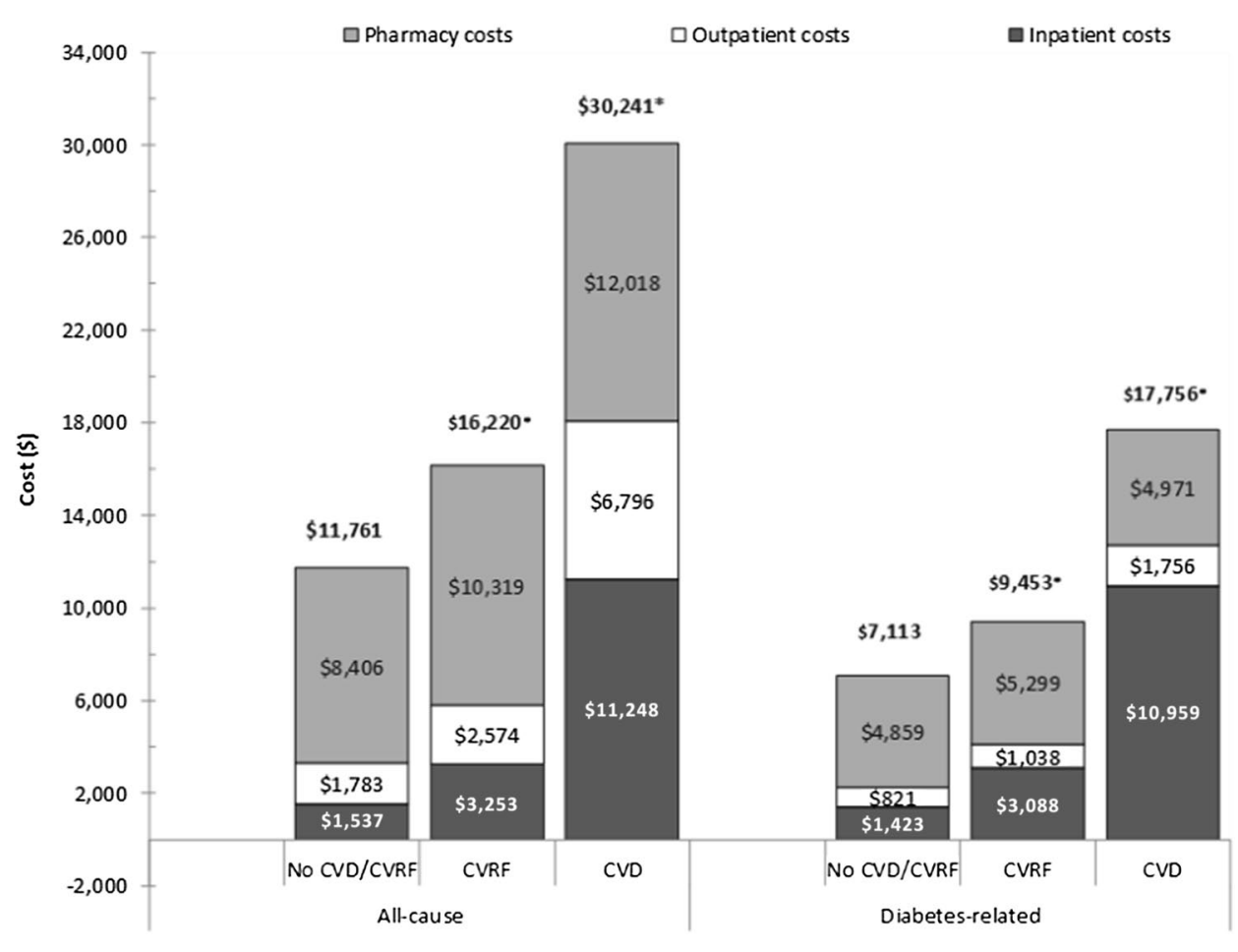

Fig. 2 Per patient per year healthcare costs at 12 months of followup (unadjusted analysis). Note: ED costs are not shown in the chart because the values are much smaller relative to those for the other categories (all-cause: $\$ 35, \$ 73$, and $\$ 178$ for the no-CVD/CVRF, CVRF, and CVD cohorts, respectively; diabetes-related: \$11, \$28, and $\$ 70$ for the no-CVD/CVRF, CVRF, and CVD cohorts, respectively). However, the total costs shown on top of the bars include ED costs. Outpatient costs include costs for outpatient visits and service

ED costs) and prescription costs (all-cause: $\$ 30,241$ for the CVD and $\$ 16,220$ for the CVRF cohorts vs. $\$ 11,761$ for the no-CVD/CVRF cohort; $P<0.05$ and $\mathrm{SMD} \geq 0.1$ for both; diabetes-related: $\$ 17,756$ for the CVD and $\$ 9453$ for the CVRF cohorts vs. $\$ 7113$ for the no-CVD/CVRF cohort; $P<0.05$ and $\mathrm{SMD} \geq 0.1$ for both).

\subsection{Multivariate Models Adjusting for Confounders}

When adjusted for confounding factors, the results for healthcare resource utilization were consistent with those obtained in the unadjusted analysis (see the ESM). The CVD and CVRF cohorts had significantly higher healthcare costs compared with the no-CVD/CVRF cohort after controlling for confounders (all $P<0.001$ ) (see Table 3 ). The estimated differences in overall all-cause and diabetes-related costs were smaller between the CVRF and the no-CVD/CVRF cohorts than between the CVD and the no-CVD/CVRF cohorts. Similar results were noted for all-cause and diabetes-related medical costs, with a larger estimated difference in medical costs for CVD vs. no CVD/CVRF compared to CVRF vs. no CVD/ CVRF. costs, whereas inpatient costs include only costs for inpatient admissions. Numbers of patients in the different cohorts were as follows: 4445 in the no-CVD/CVRF cohort, 5371 in the CVRF cohort, and 2871 in the CVD cohort. *Significant difference: $P<0.05$ and $\mathrm{SMD} \geq 0.1$ (vs. no CVD/CVRF). $C V D$ cardiovascular disease, $C V R F$ cardiovascular risk factors, $E D$ emergency department, SMD standardized mean difference

\section{Discussion}

This analysis of real-world data on the disease prevalence and economic outcomes associated with CVRF and CVD in T1D from a large US managed care program database showed that more than one in five adults with T1D have CVD, and that more than two in five have CVRF. These patients with CVD or CVRF have higher healthcare expenditures vs. those without these comorbidities.

As expected, patients in the no-CVD/CVRF cohort were younger than those in the CVRF and CVD cohorts, with fewer patients enrolled in Medicare. The most prevalent (period prevalence) CVD comorbidity was cardiac arrhythmia, with approximately half of the patients with CVD-associated comorbidities having a diagnosis code for this condition, followed by CAD/myocardial infarction/ angina. Hypercholesterolemia, hyperlipidemia, and hypertension were the most frequent CVRF identified, followed by obesity in more than one-fifth of patients.

In the initial analysis of the T1D Exchange clinic registry in the US, which included only approximately $11 \%(2861 / 25,833)$ of patients aged $\geq 50$ years, almost 


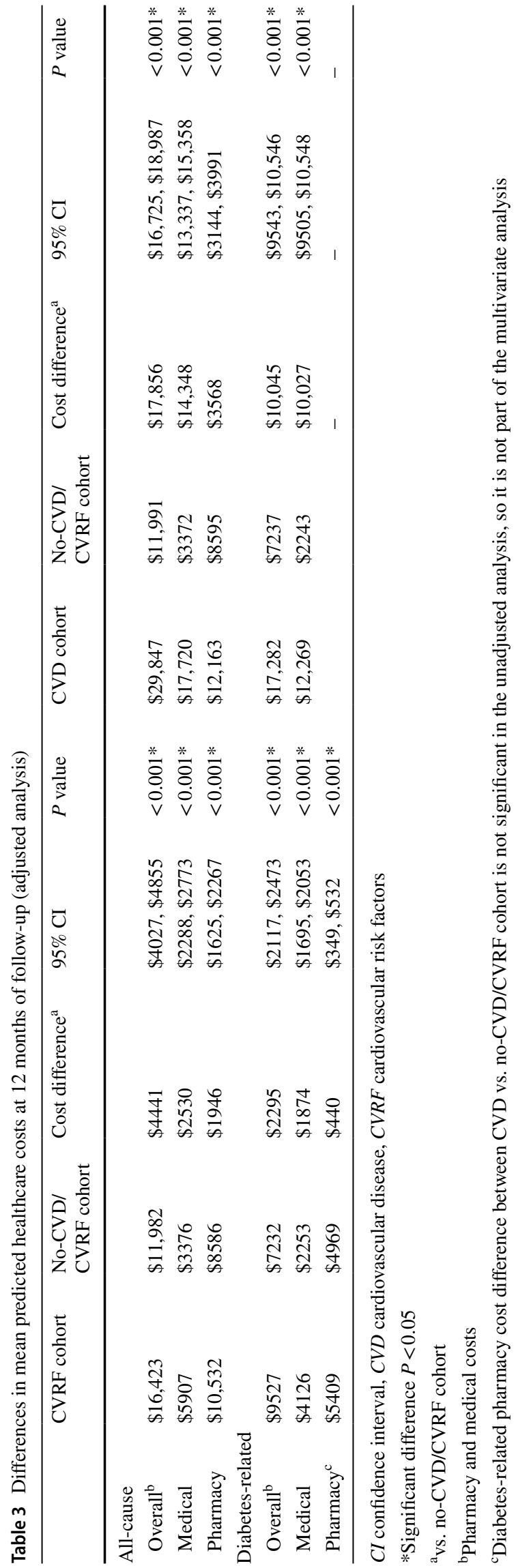

one-fourth were overweight or obese at the time of diagnosis. The frequency of kidney disease, including microand macroalbuminuria, kidney failure, glomerular filtration rate $<60 \mathrm{~mL} / \mathrm{min}$, and need for dialysis or kidney transplant, in patients aged $\geq 50$ years was $29 \%$ [17]. A subsequent data update indicated a similar percentage of obese patients specifically in this age group (29\%) [18], and a subanalysis of 9481 adult patients conducted in 2016-2017 revealed that $27 \%$ of women and $29 \%$ of men aged $\geq 50$ years were obese [19]. These rates are similar to the overall prevalence of $22 \%$ observed in our analysis. Shah et al. [18] also identified $20 \%$ and $23 \%$ of women and men, respectively, with hypertension and $45 \%$ and $56 \%$ with dyslipidemia in this age group [19], which are lower rates than the ones for the CVRF cohort in our analysis ( $67 \%$ for hypertension and $74 \%$ for dyslipidemia).

A cross-sectional study of slightly older patients with T1D ( $\geq 60$ years) compared outcomes obtained from the analyses of the T1D Exchange registry $(n=1283)$ in the US and the German/Austrian diabetes patient registries $(n=2014)$ and showed very similar rates of obesity $(25 \%)$. History of myocardial infarction and stroke was reported less frequently in the T1D Exchange registry than in its European counterparts [myocardial infarction: $6 \%$ vs. 9\%, $99 \%$ confidence interval (CI) of difference $1-5 \%$, respectively; stroke: $2 \%$ vs. $8 \%$; $99 \%$ CI 3-7\%], although no differences in body mass index were observed [20]. Our analysis revealed a higher prevalence for CVD; although our definition for CVD was much broader (including heart failure, cardiac arrhythmia, and peripheral artery disease, and not just limited to prior stroke and myocardial infarction), it must also be taken into consideration that the cohorts we analyzed were part of a broader population of T1D patients than the one in the T1D Exchange registry, which was restricted to the main diabetes care centers in the US. Therefore, there may be potential differences between these cohorts in the care provided.

The results of the unadjusted analysis showed that presence of CVD and/or CVRF had a negative impact on allcause and diabetes-related healthcare resource utilization. The magnitude of the differences vs. the no-CVD/CVRF cohort was in fact larger for the CVD than for the CVRF cohort. When adjusted for confounding factors, the results for healthcare resource utilization were consistent with those obtained in the unadjusted analysis, and the cost data reflected the differences seen in healthcare resource utilization. All-cause and diabetes-related overall costs for the CVD cohort were almost double those for the CVRF cohort. Therefore, the estimated difference in overall and medical costs vs. the no-CVD/CVRF cohort was significantly higher for the CVD cohort than for the CVRF cohort.

The present results are not entirely surprising considering previous reports in the literature. Data from the Medical 
Expenditure Panel Survey, which was conducted in the US in 2012 and involved 25,317 participants, showed that the rate of healthcare resource utilization for individuals with diabetes at a lower risk of developing CVD (i.e., patients with $\leq 1 \mathrm{CVRF}$, comprising $54 \%$ of the study population) was less than that for those at higher risk (i.e., patients with $\geq 2$ CVRF, which represented $46 \%$ of the overall study population, including $7 \%$ with $\geq 4 \mathrm{CVRF}$ ), defined as presence of hypertension and/or lack of physical exercise, smoking, and obesity [21]. The likelihood of needing to purchase/refill a prescription and having an outpatient visit was reduced by approximately $50 \%$ among patients with a favorable risk profile. This reduced use of resources translated to lower associated healthcare costs (i.e., prescription medications, hospitalizations, outpatient visits, and other expenses such as care at home and medical supplies) for patients with diabetes and an optimal CVRF profile compared to those with diabetes and a poor CVRF profile (\$6461 vs. \$9006; $P<0.001$ ) [21]. This difference of $\$ 2545$ was lower than the adjusted difference for all-cause healthcare costs between the CVRF cohort and the no-CVD/CVRF cohort $(\$ 4441,95 \%$ CI \$4027-4855; $P<0.001)$ in the present analysis, which is not unexpected given that the optimal category group in the study by Feldman et al. [21] could have the presence of one risk factor.

UnitedHealthcare is the largest provider of health insurance in the US. Therefore, the Optum ${ }^{\circledR}$ Clinformatics $^{\mathrm{TM}}$ Data Mart represents patients from all regions of the country, and the large size and regional diversity of the data should help mitigate some forms of selection bias. However, it is possible that patients insured at UnitedHealthcare may differ in demographic and healthcare-seeking behaviors from those insured by other providers or the uninsured (who represented approximately $9 \%$ of the population in 2016) [22]. This analysis was not able to assess this type of selection bias given the lack of access to data from other insurance companies or the uninsured, and there is also the possibility of selection bias due to the requirement for the patients to be continuously enrolled in the health plan for 2 years. The generalizability of the population evaluated to the broader adult population of patients with T1D may be also limited, since this database overrepresents commercially insured and Medicare-covered patients. Finally, diagnosis coding errors ultimately affect the analysis and, without a medical chart validation, it is possible that a small proportion of the patients included in the analysis may have been misclassified as having T1D. Limitations on capturing CKD and obesity as a risk factor using ICD codes may not be as comprehensive as using laboratory data, so some cases of CKD/obesity may not have been captured.

\section{Conclusion}

In summary, more than one in five adults with T1D have CVD, and approximately two in five have CVRF, with higher healthcare expenditures seen for these patients compared to those without CVD or CVRF. The management of T1D is complex and costly, and the tendency of T1D to have an earlier onset than T2D results in longer exposure to the complications of the disease in this patient population. Potentially, better management of CVD and/or related risk factors in T1D could significantly reduce the economic burden and improve outcomes for these patients.

Author contributions SE interpreted the data, reviewed the $\operatorname{draft}(\mathrm{s})$ of the manuscript, provided approval of the final version to be submitted, and is accountable for the accuracy and integrity of the manuscript. FLZ, RP, SP, MJD, and VNJ participated in the study design, interpreted the data, reviewed the draft(s) of the manuscript, provided approval of the final version to be submitted, and are accountable for the accuracy and integrity of the manuscript. SV collected and interpreted the data, reviewed the $\operatorname{draft}(\mathrm{s})$ of the manuscript, provided approval of the final version to be submitted, and is accountable for the accuracy and integrity of the manuscript.

Data availability The datasets analyzed are not publicly available because of restrictions imposed by the provider of the data $\left(\right.$ Optum $\left.{ }^{\circledR}\right)$.

\section{Compliance with Ethical Standards}

Funding This study was funded by Sanofi US, Inc. The authors received writing/editorial support in the preparation of this manuscript from Patricia Fonseca, $\mathrm{PhD}$, an employee of Excerpta Medica, funded by Sanofi US, Inc.

Conflict of interest SE is an advisory panel member for AstraZeneca, BrightSight, InPen, Lexicon, Lilly USA, MannKind, Merck, Novo Nordisk, and Sanofi, a member of the speakers' bureau of AstraZeneca, InPen, Lilly USA, MannKind, Merck, and Sanofi, and a board member of Senseonics and TeamType1. FLZ, RP, and SP are employees of Sanofi US, Inc. FLZ and RP are stock holders of Sanofi US, Inc. $\mathrm{SV}$ is an employee of Evidera, which is under contract with Sanofi US, Inc. MJD and VNJ are employees of Lexicon Pharmaceuticals, Inc. MJD is a stock options/stock holder of Lexicon Pharmaceuticals, Inc.

Open Access This article is licensed under a Creative Commons Attribution-NonCommercial 4.0 International License, which permits any non-commercial use, sharing, adaptation, distribution and reproduction in any medium or format, as long as you give appropriate credit to the original author(s) and the source, provide a link to the Creative Commons licence, and indicate if changes were made. The images or other third party material in this article are included in the article's Creative Commons licence, unless indicated otherwise in a credit line to the material. If material is not included in the article's Creative Commons licence and your intended use is not permitted by statutory regulation or exceeds the permitted use, you will need to obtain permission directly from the copyright holder. To view a copy of this licence, visit $\mathrm{http} / / /$ creativecommons.org/licenses/by-nc/4.0/. 


\section{References}

1. Lind M, Svensson AM, Kosiborod M, et al. Glycemic control and excess mortality in type 1 diabetes. $\mathrm{N}$ Engl $\mathrm{J}$ Med. 2014;371(21):1972-82.

2. McAllister DA, Read SH, Kerssens J, et al. Incidence of hospitalization for heart failure and case-fatality among 3.25 million people with and without diabetes mellitus. Circulation. 2018;138(24):2774-866.

3. de Ferranti SD, de Boer IH, Fonseca V, et al. Type 1 diabetes mellitus and cardiovascular disease: a scientific statement from the American Heart Association and American Diabetes Association. Diabetes Care. 2014;37(10):2843-63.

4. Lee SI, Patel M, Jones CM, Narendran P. Cardiovascular disease and type 1 diabetes: prevalence, prediction and management in an ageing population. Ther Adv Chronic Dis. 2015;6(6):347-74.

5. Rawshani A, Sattar N, Franzén S, et al. Excess mortality and cardiovascular disease in young adults with type 1 diabetes in relation to age at onset: a nationwide, register-based cohort study. Lancet. 2018;392(10146):477-86.

6. Livingstone SJ, Looker HC, Hothersall EJ, et al. Risk of cardiovascular disease and total mortality in adults with type 1 diabetes: Scottish registry linkage study. PLoS Med. 2012;9(10):e1001321.

7. Livingstone SJ, Levin D, Looker HC, Scottish Diabetes Research Network epidemiology group, Scottish Renal Registry, et al. Estimated life expectancy in a Scottish cohort with type 1 diabetes, 2008-2010. JAMA. 2015;313(1):37-44.

8. Dahlqvist S, Rosengren A, Gudbjörnsdottir S, et al. Risk of atrial fibrillation in people with type 1 diabetes compared with matched controls from the general population: a prospective case-control study. Lancet Diabetes Endocrinol. 2017;5(10):799-807.

9. Larsson SC, Wallin A, Håkansson N, Stackelberg O, Bäck M, Wolk A. Type 1 and type 2 diabetes mellitus and incidence of seven cardiovascular diseases. Int J Cardiol. 2018;262:66-70.

10. Secrest AM, Becker DJ, Kelsey SF, Laporte RE, Orchard TJ. Cause-specific mortality trends in a large population-based cohort with long-standing childhood-onset type 1 diabetes. Diabetes. 2010;59(12):3216-22.

11. Optum. Clinformatics Data Mart. https://www.optum.com/conte nt/dam/optum/resources/productSheets/Clinformatics_for_Data_ Mart.pdf. Accessed 15 Jul 2019.
12. Klompas M, Eggleston E, McVetta J, Lazarus R, Li L, Platt R. Automated detection and classification of type 1 versus type 2 diabetes using electronic health record data. Diabetes Care. 2013;36(4):914-21.

13. Schroeder EB, Donahoo WT, Goodrich GK, Raebel MA. Validation of an algorithm for identifying type 1 diabetes in adults based on electronic health record data. Pharmacoepidemiol Drug Saf. 2018;27(10):1053-9.

14. World Health Organization. International classification of diseases. ICD-11: classifying disease to map the way we live and die. https://www.who.int/health-topics/international-classifica tion-of-diseases. Accessed 15 Jul 2019.

15. Bureau of Labor Statistics. Consumer Price Index. https://www. bls.gov/cpi/. Accessed 15 Jul 2019.

16. Long JS, Freese J. Regression models for categorical dependent variables using Stata. 3rd ed. College Station: Stata Press; 2014.

17. Beck RW, Tamborlane WW, Bergenstal RM, Miller KM, DuBose SN, Hall CA, T1D Exchange Clinic Network. The T1D Exchange clinic registry. J Clin Endocrinol Metab. 2012;97(12):4383-9.

18. Miller KM, Foster NC, Beck RW, et al. Current state of type 1 diabetes treatment in the US: updated data from the T1D Exchange clinic registry. Diabetes Care. 2015;38(6):971-8.

19. Shah VN, Wu M, Polsky S, et al. Gender differences in diabetes self-care in adults with type 1 diabetes: findings from the T1D Exchange clinic registry. J Diabetes Complications. 2018;32(10):961-5.

20. Weinstock RS, Schütz-Fuhrmann I, Connor CG, T1D Exchange Clinic Network; DPV Initiative, et al. Type 1 diabetes in older adults: comparing treatments and chronic complications in the United States T1D Exchange and the German/Austrian DPV registries. Diabetes Res Clin Pract. 2016;122:28-37.

21. Feldman DI, Valero-Elizondo J, Salami JA, et al. Favorable cardiovascular risk factor profile is associated with lower healthcare expenditure and resource utilization among adults with diabetes mellitus free of established cardiovascular disease: 2012 Medical Expenditure Panel Survey (MEPS). Atherosclerosis. 2017;258:79-83.

22. United States Census Bureau. Health insurance coverage in the United States. 2016. https://www.census.gov/library/publicatio ns/2017/demo/p60-260.html. Accessed 15 Jul 2019. 